\title{
Expression of Canavalia gladiata lectin in leukemic cells
}

\author{
Laija. S. Nair ${ }^{1 *}$, Mahesh. $\mathbf{S}^{2}$, Smitha L. $\mathbf{S}^{3}$, Sujathan. $\mathrm{K}^{3}$ and Remani. $\mathbf{P}^{3}$
}

${ }^{1}$ Department of Botany, Government Sanskrit College, Pattambi, Palakkad, Kerala, India

${ }^{2}$ Department of Botany, Christian College, Kattakada, Thiruvananthapuram, Kerala, India

${ }^{3}$ Division of Cancer Research, Regional Cancer Centre, Thiruvananthapuram, Kerala, India

\begin{abstract}
In this study an attempt was made to find out new tissue specific lectin from the seeds of Canavalia gladiata and to investigate whether this could be used as biological markers in haematological malignancies. Lectin binding studies showed that Lymphoid leukemias such as ALL (Acute Lymphocytic Leukemia) and CLL (Chronic Lymphocytic Leukemia) cells did not show any reactivity with CGL (Canavalia gladiata lectin). Blast cells of myeloid leukemias showed varying intensities of staining. Kruskal Wallis One Way ANOVA showed significance in expression of the lectin with different types of Acute Myeloid Leukemia. Lectin cytochemical studies in leukemia revealed its usefulness in differentiating lymphoid leukemias from myeloid leukemias.
\end{abstract}

Keywords: Canavalia gladiata lectin; Lymphocytic leukemia; Myeloid Leukemia

Abbreviatons: CGL : Canavalia Gladiata Lectin; ALL: Acute Lymphocytic Leukemia; CLL: Chronic Lymphocytic Leukemia; AML: Acute Myeloid Leukemia; CML: Chronic Myeloid Leukemia; HRP : Horse Radish Peroxidase

\section{Introduction}

Leukemia is an unregulated proliferation of haematopoietic tissue that progressively displaces normal blood cell elements. Leukemias are traditionally classified by the predominant cell type involved and the degree of maturity or immaturity that characterizes the peripheral blood cell pattern.

Plant lectins are of great use in the detection of the complex carbohydrates of the cell membrane. Lectins are valuable cyto and histological tools for the localization of defined carbohydrate determinants. Horse Radish Peroxidase labelled lectins were used for the histochemical distinction of glycolipids with various terminal hexoses in different storage diseases as well as for the demonstration of differences in the composition of cell surface and intercellular glycoproteins in tissues at various stages of differentiation, in malignancy and in functional subset of cells.

In this study Horseradish peroxidase conjugated Canavalia gladiata lectin was used to evaluate its binding property to different types of leukemic cells and to investigate whether this lectin could be used for the typing of leukemias.

\section{Materials and Methods}

\section{Conjugation of lectins to horse radish peroxidsae}

Conjugation of lectin was carried in two steps, activation of Horse Radish Peroxidase (HRP) and then conjugation.

Activation of HRP: $10 \mathrm{mg}$ of Horse Radish Peroxidase type VI (Sigma, St. Louis. USA) was dissolved in $2 \mathrm{ml}$ of $2.5 \%$ sodium bicarbonate solution. $20 \mu \mathrm{l}$ of $1 \%$ Fluorodinitrobenzene (FDNB) in ethanol was added and mixed gently for one hour at room temperature. $1 \mathrm{ml}$ of $0.06 \mathrm{M}$ sodium periodate was added and mixed gently for 30 minutes. Two drops of ethylene glycol was then added to it and mixed gently for one hour at room temperature. The activated peroxidase was then desalted on Sephadex G-25 equilibrated with bicarbonate buffer ( $\mathrm{pH}$ 9.5). The bicarbonate buffer was prepared by dissolving anhydrous sodium carbonate $(2.756 \mathrm{~g} / \mathrm{l})$ and sodium bicarbonate $(6.216 \mathrm{~g} / \mathrm{l})$ in $1000 \mathrm{ml}$ distilled water and the $\mathrm{pH}$ was adjusted to 9.5 . Two grams of Sephadex G-25 was soaked in $15 \mathrm{ml}$ of distilled water on the day before activation. The Sephadex was packed in an $8 \mathrm{ml}$ column up to $2 / 3$ of its length and was washed thoroughly with bicarbonate buffer ( $\mathrm{pH} 9.5$ ). The HRP solution was allowed to run the column and the effluents were collected in $2 \mathrm{ml}$ fractions. The optical density of the fractions was measured at $280 \mathrm{~nm}$ and the fractions with higher peaks were pooled and refrigerated for conjugation.

Conjugation: $10 \mathrm{mg}$ of the lectin was dissolved in bicarbonate buffer ( $\mathrm{pH} 9.5$ ) and sufficient amount of mannose to get a $20 \mathrm{mM}$ concentration was added and kept for one hour at room temperature. To this the activated Horse Radish Peroxidase (HRP) was added and mixed gently for three hours at room temperature. $10 \mathrm{mg}$ of sodium borohydride was then added and left for one hour at room temperature. Now the $\mathrm{pH}$ was lowered to 6.9 by adding $1 \mathrm{~N} \mathrm{HCl}$ and kept overnight in the refrigerator at $4^{\circ} \mathrm{C}$.

One and a half gram of Sephadex G-200 was soaked in distilled water one day before the day of conjugation and on the day it was packed in a long column $(75 \times 0.5 \mathrm{~cm})$ and washed with Phosphate Buffered Saline (PBS) ( $\mathrm{pH}$ 7.2). The lectin HRP mixture was then allowed to run through the column and the effluents were collected in $2 \mathrm{ml}$ fractions in 20 tubes. The optical density of the effluents was measured at $280 \mathrm{~nm}$. Fraction with peak absorbance was kept at $4^{\circ} \mathrm{C}$ until used for staining.

\section{Smears used for the study}

Peripheral blood and bone marrow smears from 180 leukemic patients, from the clinic of Regional Cancer Centre were used for the study. Peripheral blood and bone marrow smears from 15 patients

*Corresponding author: Laija. S. Nair, Department of Botany, Government Sanskrit College, Pattambi, Palakkad, Kerala, India, E-mail: laijamahesh@gmail.com

Received December 10, 2010; Accepted February 14, 2011; Published February 18, 2011

Citation: Nair LS, Mahesh S, Smitha LS, Sujathan K, Remani P (2011) Expression of Canavalia gladiata lectin in leukemic cells. J Cancer Sci Ther 3: 088-091. doi:10.4172/1948-5956.1000066

Copyright: ( 2011 Nair LS, et al. This is an open-access article distributed under the terms of the Creative Commons Attribution License, which permits unrestricted use, distribution, and reproduction in any medium, provided the original author and source are credited. 
Citation: Nair LS, Mahesh S, Smitha LS, Sujathan K, Remani P (2011) Expression of Canavalia gladiata lectin in leukemic cells. J Cancer Sci Ther 3: 088-091. doi:10.4172/1948-5956.1000066

undergoing investigation for various complaints but reported as normal were used as controls. The number of cases of each type of leukemia used for the study is given in Table 1 . Out of the 180 leukemic patients, 114 were males $(63.3 \%)$ and $66(36.7 \%)$ were females (Table 1$)$. The male to female ratio was 1.07:1.The age of the patients included in this study varied from 1 to 66 years.

\section{Staining procedure}

The peripheral blood and bone marrow smears were fixed in methanol for 10minutes and rinsed in three changes of absolute alcohol for 10 minutes in each change. The slides were then immersed in 3\% hydrogen peroxide in methanol for 30 minutes to block the endogenous peroxidase activity [1]. The smears were then rinsed in three changes of PBS (pH7.4) for 5 minutes in each change. Incubating with bovine serum albumin for 30 minutes at room temperature blocked the non specific binding. After removing the bovine serum albumin by blotting, the smears were incubated with the respective lectin conjugate overnight at $4^{\circ} \mathrm{C}$. The smears were then rinsed in three changes of Phosphate Buffered Saline and stained in 30\% 3'-3'-diaminobenzidine (DAB) containing hydrogen peroxide for 5 minutes. It was then washed in three changes of distilled water and counter stained in Harris haematoxylin for 2 minutes. The slides were then dehydrated in ascending grades of alcohol and mounted in DPX. Smears from each case, untreated with the lectin conjugate, but treated with DAB solution for 5 minutes served as the negative control.

The slides were scored under light microscope and recorded by a visual assessment of the intensity of the brownish reaction product on the blast cells. 100 blast cells were studied in each sample. The expression patterns of various lectins studied were blindly evaluated by two investigators independently without knowing the haematology of the sample. Lectin binding was graded as negative, mild and intense based on the intensity of staining in the blast cells. Staining grades were converted into numerical scores, negative staining was categorized as 1 , mild as 2, intense as 3 for analysis. For assessing the staining pattern

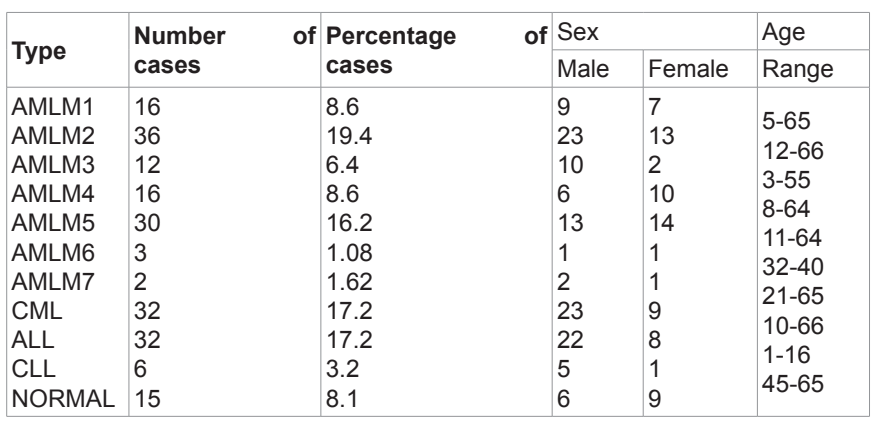

Table 1: Distribution of Cases.

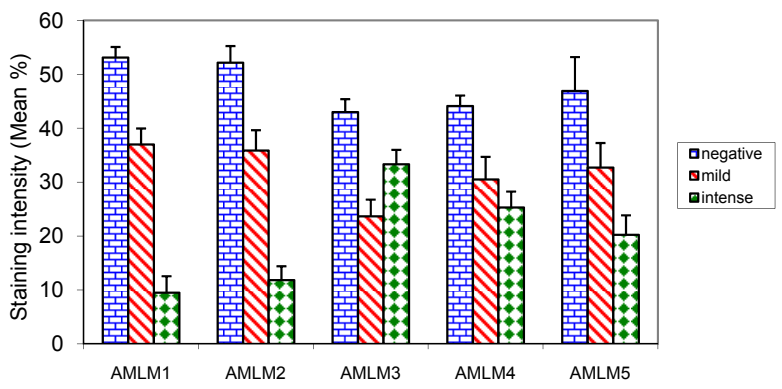

Figure 1: Bar diagram showing CGL binding intensities in different types of AML.
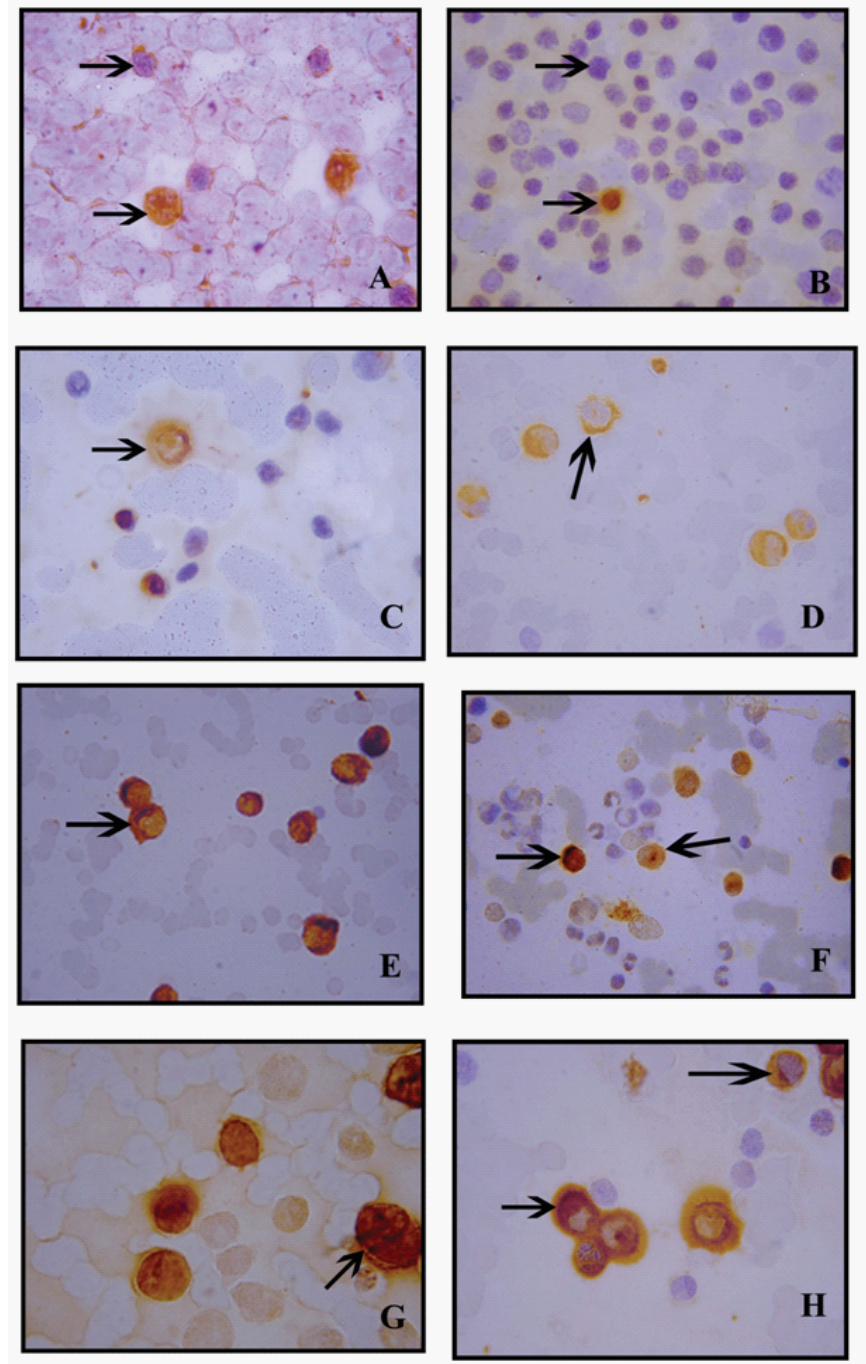

Figure 2:

A. Lymphoblasts showing negative staining and promyelocytes showing positive staining in Chronic lymphocytic leukemia.

B. Lymphoblasts showing negative staining and promyelocyte showing positive staining in Acute Lymphocytic Leukemia.

C. Acute Myeloid Leukemia M1 cells showing mild staining with Canavalia gladiata lectin.

D. Acute Myeloid Leukemia M2 cells showing mild staining with Canavalia gladiata lectin.

E. Acute Myeloid Leukemia M3 cells showing intense cap like staining pattern with Canavalia gladiata lectin.

F. Acute Myeloid Leukemia M4 cells showing moderate and intense staining with Canavalia gladiata lectin.

G. Myelomonocytic leukemia (AMLM5) cells showing intense staining with Canavalia gladiata lectin.

$\mathrm{H}$. Chronic Myeloid Leukemia cells showing intense membrane staining with Canavalia gladiata lectin.

of each lectin, both percentage of positivity and intensity of staining were recorded. Therefore, to nullify the effect of either the percentage of positivity or staining intensity the product of these two was taken for the statistical analysis. Considering the lower sample size, AMLM6 and AMLM7 were excluded from statistical analysis. The differential expression of lectin binding was analyzed statitistically using Kruskal Wallis test. 


\section{Result}

In the present study the interaction of the lectin with normal peripheral blood lymphocytes and various types of leukemic blast cells was investigated. In normal smears, cells of the neutrophilic series showed mild positivity with CGL. Polymorphs showed strong positivity and RBCs showed weak binding with the lectin whereas lymphocytes, platelets and eosinophils showed negative staining. AML and CML cells stained positively and the lectin exhibited negative binding to the lymphoblasts in both acute lymphocytic leukemia (Figure 2B) and chronic lymphocytic leukemia (Figure 2A). In AMLM1 myeloblasts and promyelocytes showed mild staining (Figure 2C). Monoblasts showed faint positivity and myeloblasts showed granular positivity in AMLM4 (Figure 2F). Neutrophils of Acute myeloid leukemia showed diffuse positivity. Neutrophils, myelocytes, promyelocytes and metamyelocytes are intensely stained with CML cases. Majority of the lectin positive blast cells were unable to take up haematoxylin and the nucleus was not clearly visible in majority of the blast cells. Some of the blast cells showed a cap like staining pattern. Histiocytes and Auer rods are found to be strongly positive for CGL. Neoplastic lymphoid cells of ALL and CLL did not showed binding with the lectin. Twelve cases of AMLM5 showed mild staining with CGL (Figure 2G). The lectin exhibited a mild membrane staining with the AMLM1 and AMLM2 (Figure 2C and 2D). AMLM3 cells showed characteristic cap like staining pattern (Figure 2E) and moderate to intense staining of AMLM4 cells was exhibited by CGL (Figure 2F). Cells of myelomonocytic leukemia (AMLM5) showed intense staining with CGL (Figure $2 \mathrm{G}$ ). The positively stained AML and CML cases were included in the analysis and mean value of the expression pattern of the lectins were found to vary.

The percentage of cells showing mild grade of staining was less in CML. The mean percentage of negatively stained blast cells with CGL in CML was 24.75 and intensely stained was 57.93. In general the cells of Chronic myeloid leukemia showed intense membrane staining with CGL. The percentage of blast cells showing negative as well as mild staining were shown to decrease from AMLM1 to AMLM4 (Figure 1). A sharp increase in the number of cells showing intense staining with CGL was observed from AMLM1 to AMLM4. About 20\% of blast cells stained intensely with CGL in AMLM5 (Table 2). Kruskal Wallis One Way Anova showed significance for the expression of CGL in various types of Acute myeloid leukemia (Table 3).

\section{Discussion}

Leukemias are clinically and biologically heterogenous. Classification of various leukemias according to the cellular origin is of diagnostic and therapeutic importance. However such a classification

\begin{tabular}{|l|l|l|l|}
\hline \multirow{2}{*}{ TYPE } & \multicolumn{3}{l|}{ STAINING INTENSIY } \\
\cline { 2 - 4 } & Negative & Mild & Intense \\
\hline AMLM1 & 53.12 & 37.00 & 9.50 \\
AMLM2 & 52.16 & 35.88 & 11.83 \\
AMLM3 & 43.00 & 23.66 & 33.33 \\
AMLM4 & 44.12 & 30.50 & 25.30 \\
AMLM5 & 46.93 & 32.73 & 20.40 \\
\hline
\end{tabular}

Table 2: Percentage of staining intensity of CGL in Acute Myeloid Leukemia.

\begin{tabular}{|l|l|l|l|}
\hline TYPE & MEAN \pm S.D & MEDIAN & Kruskal Wallis test \\
\hline AMLM1 & $155.62 \pm 4.78$ & 156 & \\
AMLM2 & $159.44 \pm 4.62$ & 159.5 & Chi square $=85.850$ \\
AMLM3 & $190.33 \pm 4.03$ & 190 & $\mathrm{P}<=0.0001^{*}$ \\
AMLM4 & $181.25 \pm 2.76$ & 180.5 & \\
AMLM5 & $163.4 \pm 9.74$ & 172 & \\
\hline
\end{tabular}

S.D $=$ Standard deviation * significant

Table 3: Mean Median and Kruskal Wallis One way ANOVA of the expression index of $C G L$ in various types of AML. is hampered by the scarcity of cell markers that can be attributed to lymphoid or myeloid subpopulations at specific stages of their differentiation.

Lectins from various sources bind to different but very specific sequence of sugars, thus providing a valuable tool for the study of glycoproteins. There is little information available on the binding pattern of lectins in normal and abnormal haematopoietic cells $[2,3]$. Available reports include work carried out mostly with the aid of immunofluorescent techniques, where the morphology of the cell is difficult to determine. In the present study the interaction of mannose specific lectin with normal peripheral blood lymphocytes and various types of leukemic blast cells was investigated. CGL exhibited strong binding to the cytoplasm and cell membrane of tumour cells. Parmley et al. [4] has also reported the same type of binding pattern in these cells using Con A. Monoblasts showed moderate staining with the lectin. The lectin used in the present study showed cap like staining pattern for certain blast cells. Patching or capping appears to be a biochemically distinguishable process. Patching and capping induced by lectin, is thus, very similar to that caused by anti immunoglobulins and other antibodies to lymphocyte surface constituents.

Jacobson et al. [5] have observed the binding of fluorescinated Con A in ALL cells; whereas in the present study CGL, a mannose specific lectin isolated from the same genera (Canavalia) failed to bind ALL cells. Such differences may be probably due to difficulty in determining the exact morphology of cells in immunofluorescent techniques. Another reason may be the use of different types of fixative. It is an established fact that even a change in the fixative used can lead to variations in the results of lectin histochemistry [6].

Plant lectins interact with cells by binding to specific cell surface saccharide structures. The absence of lectin binding in the CLL lymphocytes suggests that these cells might have a defect in the synthesis of complex oligosaccharides. This study confirms the earlier reports that showed reduced receptor sites for the plant lectin, Con A [7] and Agaricus bisporus haemagglutinin [8] on the CLL lymphocytes. Reisner et al. [9] have found that the lymphocytes of the majority of Chronic Lymphocytic Leukemia patients did not bind the lectin.

Lectin binding to the surface of AML blast cells among patients showed considerable variability. In all types of myeloid leukemias there were blast cells not bound by the lectins. The percentage of blast cells that bound the lectins was lower than the total percentage of blastic cells. In AMLM1 myeloblasts showed mild staining with the lectin when compared with AMLM2 and AMLM3 myeloblasts. Cytoplasmic granular staining of myeloblasts similar to peroxidase staining was observed with the lectin. But unlike peroxidase that showed negative staining with monoblasts, CGL showed mild staining. Since in AMLM5 more than $80 \%$ of cells are monocytic, statistical analysis of lectin binding in AMLM5 blasts showed 20\% intensely stained cells with CGL.

The lymphocytes of the chronic lymphocytic patients and acute lymphocytic patients did not bind to the lectin and in this respect were similar to normal peripheral blood lymphocytes. In addition the lectinbinding data suggests that CLL and ALL membrane may be deficient in mannose ligands. The cases of lymphocytic leukemia in which lectin positive blasts were absent may reflect other pathways of differentiation, where masking of lectin receptors may occur at an earlier stage. Since the lectins bind to myeloblasts and monoblasts it can be used to differentiate myeloblastic leukemia from lymphocytic leukemia. The presence of lectin receptors on the blastic cells could be of considerable importance because they may serve as targets for drug binding and enable selective destruction of pathogenic cells. 
Citation: Nair LS, Mahesh S, Smitha LS, Sujathan K, Remani P (2011) Expression of Canavalia gladiata lectin in leukemic cells. J Cancer Sci Ther 3: 088-091. doi:10.4172/1948-5956.1000066

\section{References}

1. Streefkerk JG (1972) Inhibition of erythrocyte pseudoperoxidase activity by treatment with hydrogen peroxide following methanol. J Histochem Cytochem 20: 829-831.

2. Delwel R, Touw I, Bot F, Lowenberg B (1986) Fucose binding lectin for characterizing acute myeloid leukemia progenitor cells. Blood 68: 41-45.

3. Gabius HJ, Vehmeyer K, Gabius S, Nagel GA (1988) Clinical application of various plant and endogenous lectins to leukemia. Blut 56: 147-152.

4. Parmley TR, Martin BJ, Spicer SS (1973) Staining of blood cell surfaces with a Lectin- Horseradish Peroxidase method. J Histochem Cytochem 21: 912-922.

5. Jacobson W, Stoddart RW, Collins RD (1980) Lectin staining of carbohydrates of haemic cells. II. The cells of normal lymphoid origin, of lymphatic leukemias and related diseases. Histopathology 4: 491-500.

6. Walker RA (1985) Ulex europaeus l--peroxidase as a marker of vascula endothelium: its application in routine histopathology. J Pathol 146: 123-127.

7. Novogrodsky A, Biniamnov M, Ramot B, Katchalski E (1972) Binding of Concanavalin $A$ to rat, normal human, and chronic lymphatic leukemia lymphocytes. Blood 40: 311-316.

8. Presant CA, Kornfeld S (1972) Characterization of the cell surface receptor for the Agaricus bisporus hamagglutinin. J Biol Chem 247: 6937-6945.

9. Reisner Y, Biniaminov M, Rosenthal E, Sharon N, Ramot B (1979) Interaction of peanut agglutinin with normal human lymphocytes and with leukemic cells. Proc Natl Acad Sci USA 76: 447-451. 